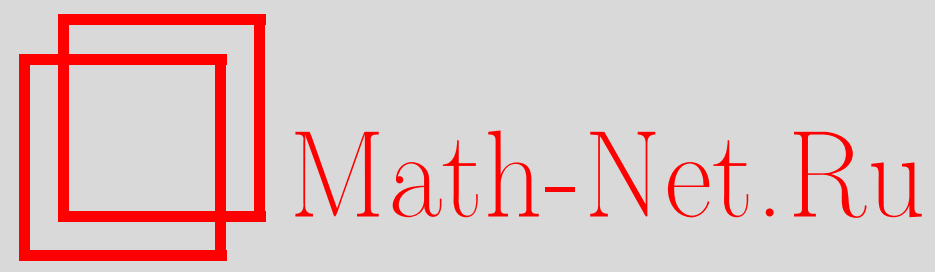

Г. К. Седов, Стойкость ГОСТ Р 34.11-2012 к атаке поиска прообраза и к атаке поиска коллизий, Матем. вопр. криптогр., 2015, том 6, выпуск 2, 79-98

DOI: https://doi.org/10.4213/mvk147

Использование Общероссийского математического портала Math-Net.Ru подразумевает, что вы прочитали и согласны с пользовательским соглашением

http://www . mathnet.ru/rus/agreement

Параметры загрузки:

IP : 34.227 .88 .159

26 апреля 2023 г., $17: 41: 13$

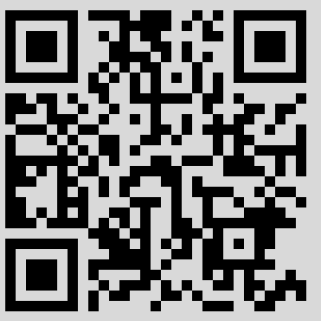


УДК: 519.719 .2

\title{
Стойкость ГОСТ Р 34.11-2012 \\ к атаке поиска прообраза \\ и к атаке поиска коллизий
}

\author{
Г.К.Седов
}

Московский государственный университет имени М. В. Ломоносова, Москва

Получено 18.XI.2014

В январе 2013 года национальный стандарт Российской Федерации, ГОСТ Р 34.11-94 определяющий алгоритм и процедуру вычисления хэшфункции, был заменен на новый ГОСТ Р 34.11-2012. В качестве нового стандарта было утверждено семейство хэш-функций Стрибог. В данной работе семейство хэш-функций Стрибог рассмотрено с позиций математической криптографии; доказана его стойкость к атаке поиска прообраза и к атаке поиска коллизий.

Ключевые слова: хэш-функции, математическая криптография, Стрибог, ГОСТ Р 34.11-2012

The security of GOST R 34.11-2012 against preimage and collision attacks

G. K. Sedov

Lomonosov Moscow State University, Moscow

Abstract. In January 2013 the National standard of the Russian Federation GOST R 34.11-94 defining the algorithm and computational procedure for hash function was replaced by GOST R 34.11-2012. A family of hash functions Streebog was approved as a new standard. We analyse the family Streebog from the mathematical cryptography viewpoint and prove that it is secure against preimage and collision attacks.

Key words: hash functions, mathematical cryptography, Streebog, GOST R 34.11-2012

Citation: Mathematical Aspects of Cryptography, 2015, vol. 6, no. 2, pp. 79-98 (Russian). 


\section{1. Введение}

ГОСТ Р 34.11-2012 [1], или Стрибог, - хэш-функции, принятые 1 января 2013 года в качестве национального стандарта Российской Федерации, определяющего алгоритм и процедуру вычисления хэш-функции. Старый стандарт был заменен после успешной атаки Ф. Менделя (F. Mendel) и др., понизившей сложность построения коллизий в $2^{23}$ раз [2].

Новый стандарт вызвал повышенное внимание криптографического научного сообщества, и в 2013 году появилось несколько работ, посвященных криптоанализу Стрибога. В частности, в работе Р. АльТави (R. AlTawy) и др. [3] описывается процедура построения коллизий и почти коллизий со свободным стартом для сокращенного до 4.5, 5.5 и 7.75 раундов внутреннего шифра функции сжатия, используемой в хэш-функции Стрибог. В этой работе внутренний шифр рассматривался как функция сжатия.

Несколько позже появилась статья 3. Ванг (Z. Wang) и др. [4], связанная с построением коллизий для сокращенной до 9.5 раундов функции сжатия со сложностью по времени $2^{176}$ и необходимым количеством памяти $2^{128}$ байт. Также в этой работе представлен метод построения $k$-коллизий для 512 битной версии функции Стрибог.

В дальнейшем P. АльТави (R. AlТawy) и Амр M. Юссуф (Amr M. Youssef) опубликовали статьи по исследованию стойкости функции Стрибог к нахождению прообраза [7] и по исследованию злонамеренного (malicious) хэширования с помощью функции Стрибог [8]. В [7] был получен псевдопрообраз для сокращенной до 5 раундов функции сжатия со сложностью $2^{448}$ по времени и с необходимым количеством памяти $2^{64}$ бит. В работе [8] авторы исследуют функцию Стрибог с измененными раундовыми константами и числом раундов функции сжатия, равным 12, и для такого варианта функции Стрибог описывают двухблоковую коллизию.

Также известна работа Бинкге Ма (Bingke Ma) и др. [9], в которой авторы предлагают атаки, связанные с нахождением прообраза и построением коллизий, на сокращенные версии хэш-функции Стрибог. В этой работе авторы предлагают улучшенный алгоритм построения прообраза для сокращенной до 6 раундов функции Стрибог, а также улучшенный алгоритм построения коллизий для сокращенной до 7,5 раундов функции сжатия Стрибог.

Указанные выше работы по исследованию хэш-функции Стрибог посвящены построению криптографических атак на нее, и, насколько нам известно, в открытой печати нет работ, посвященных обоснованию криптографической стойкости данной хэш-функции. 
В январе 2009 года Е. Додис (Е. Dodis), Т. Ристенпарт (Т. Ristenpart) и Т. Шримптон (T. Shrimpton) [5] вводят для функций общего вида понятие стойкости к атаке на прообраз (preimage awareness) и доказывают стойкость в этом смысле некоторых функций сжатия, а также итеративной схемы Меркля-Дамгарда, используя модель идеального шифра.

В нашей работе рассматривается семейство хэш-функций Стрибог в модели идеального шифра и доказывается его стойкость к атаке поиска коллизий и стойкость к атаке поиска прообраза в определениях работы [5].

\section{2. Определения, обозначения и известные результаты}

\section{1. Определения}

Обозначения. Для двух строк $x$ и $y$ будем обозначать через $x \| y$ их конкатенацию, а записью $x^{n}$ будем обозначать конкатенацию $n$ экземпляров вектора $x$. Для некоторого множества $X$ запись $X \leftarrow x$ обозначает добавление элемента $x$ в множество $X$, а запись $y \stackrel{\$}{\longleftarrow}$ означает, что в переменную $y$ следует занести случайно выбранное значение из множества $X$. Если множество $X$ конечно, то будем рассматривать равномерное распределение, а если $X=\{0,1\}^{\infty}$, то будем использовать такое распределение, что для любого подмножества

$$
\begin{aligned}
Y_{i_{1}, i_{2}, \ldots, i_{m}}^{\sigma_{1}, \sigma_{2}, \ldots, \sigma_{m}}=\left\{y=\left(y_{1}, y_{2}, \ldots, y_{n}, \ldots\right)\right. & \in\{0,1\}^{\infty} \mid \\
& \left.y_{i_{k}}=\sigma_{k}, \sigma_{k} \in\{0,1\}, k=1, \ldots, m\right\}
\end{aligned}
$$

вероятность того, что $Y_{i_{1}, i_{2}, \ldots, i_{m}}^{\sigma_{1}, \sigma_{2}, \ldots, \sigma_{m}} \ni \alpha \stackrel{\$}{\longleftarrow}\{0,1\}^{\infty}$, равна $\frac{1}{2^{m}}$. Для строки $x$ будем обозначать через $|x|$ ее длину. Записью $m_{1}, m_{2}, \ldots, m_{k} \stackrel{n}{\leftarrow} M$ будем обозначать разбиение строки $M$ длины $k \cdot n$ на подстроки длины $n$.

В дальнейшем при построении моделей защищенности нам потребуется также определение достаточно малой функции. Функция $\mu: \mathbb{N} \rightarrow \mathbb{R}$ называется достаточно малой, если для любого полинома $p(\cdot)$ существуют такие $N \in \mathbb{N}$ и $c \in \mathbb{R}$, что для любого $n>N$

$$
|\mu(n)|<\frac{c}{p(n)} .
$$

Вероятностные машины Тьюринга (МТ). Определим вероятностную машину Тьюринга следующим образом. Пусть помимо обычной ленты с входным словом $x$, доступной машине Тьюринга $M$ и на чтение, и на запись, имеется дополнительная лента, содержащая строку $r \stackrel{\$}{\$}\{0,1\}^{\infty}$ 
и доступная $M$ только на чтение. Читающая головка данной ленты в начальный момент времени стоит на первом символе этой строки.

Для машины Тьюринга $M$ кроме основного алфавита входа $M_{A}$ определим некоторый дополнительный алфавит $M_{I}$, который будем называть алфавитом интерфейсов. Будем считать, что для каждого $i_{k} \in M_{I}$ машина $M$ на слове $i_{k}|| a$, где $a \in M_{A}$, моделирует работу некоторой машины Тьюринга $M_{i_{k}}$ на слове $a$. Каждый символ $i$ из алфавита $M_{I}$, а также MT, моделируемую $M$ на слове $a$, где $a \in M_{A}$, будем называть интерфейсом.

Взаимодействие МТ $A$ и МТ $B$ будем описывать следующим образом. Будем считать, что помимо своих лент у $A$ и $B$ есть общая лента и каждая из МТ имеет к ней доступ на чтение и на запись. Машина $A$ производит вычисления на своем входном слове $x_{A}$, затем записывает некоторое сообщение $m_{1}$ на общую ленту и переходит в специальное состояние ожидания, запуская тем самым машину $B$. Машина $B$ производит некоторые вычисления над своим входным словом $x_{B}$ и сообщением $m_{1}$, записывает на общую ленту сообщение $m_{2}$ и переходит в состояние ожидания, запуская МТ $A$. Если на входных словах $x_{A}, x_{B}$ МТ $A$ и $B$ останавливаются и выдают сообщения $y_{A}$ и $y_{B}$ соответственно, то пара $y_{A}, y_{B}$ называется результатом взаймодействия машин $A$ и $B$.

Если во взаимодействии машин $A$ и $B$ необходимо выделить машину $A$ как основную, а машину $B$ как зависимую, взаимодействие машин $A$ и $B$ называется доступом машины $A \kappa$ машине $B$, или считается, что МТ $A$ может делать запросы к МТ $B$. Иногда в таком случае машина $B$ называется оракулом. Для МТ $A$ формула $x \Leftarrow A$ обозначает запись вывода $A$ в переменную $x$, а формула $x \stackrel{\$}{\rightleftarrows} A-$ запись вывода в переменную при условии, что МТ $A$ может быть вероятностной. Возможность доступа некоторой МТ $A$ к МТ $P_{1}, P_{2}, \ldots, P_{n}, \ldots$ обозначается $A^{P_{1}, P_{2}, \ldots, P_{n}, \ldots}$. Будем писать $A \Rightarrow a$ для обозначения события, при котором МТ $A$ вывела значение $a$. Под словами $M T B$ запускает $M T A$, перенаправляя запросы $M T A \kappa$ оракулу $P_{A}$ на вход оракулу $P_{B}$, подразумевается следующий процесс: МТ $B$ имеет доступ к машине $A$, у которой общая лента с машиной $P_{A}$ заменена на общую ленту с машиной $P_{B}$. Эффективным по параметру $k$ алгоритмом называется алгоритм, выполняющийся за полиномиальное по параметру $k$ время. Под словами «алгоритм» и «процедура» в дальнейшем понимается некоторая машина Тьюринга, возможно вероятностная.

Для алгоритма $f$, получающего на вход строку из некоторого непустого множества $\operatorname{Dom} \subseteq\{0,1\}^{*}$, определяется $\operatorname{Time}(f, m)$ как максимальное время работы алгоритма $f(x)$ для любого такого входа $x \in D o m$, что $|x| \leqslant m$. Ес- 
ли второй аргумент опускается, то Time $(f)$ рассматривается как максимальное время работы для всех возможных входов $x \in D o m$. Под обозначением $\operatorname{Time}(f(x))$ понимается время работы $f$ на строке $x \in D$ om. При подсчете времени работы МТ $A$ время ожидания ответа от оракула $B$ будем считать одним тактом работы МТ $A$.

Идеальные примитивы. Под идеальным примитивом подразумевается некоторая (возможно, вероятностная) машина Тьюринга. Данное определение используется, чтобы подчеркнуть использование этой машины Тьюринга в построении более сложных конструкций (см. [5, с. 7]).

Для фиксированных множеств Dom, Rng случайным оракулом $\mathcal{F}_{D o m, R n g}$ называется идеальный примитив, действующий следующим образом. При первом запросе у оракула $\mathcal{F}_{D o m, R n g}$ некоторого значения $x_{i} \in D o m$ он сопоставляет ему некоторое случайно выбранное значение $y_{i} \in R n g$ и возвращает $y_{i}$. При повторном запросе $x_{i}$ возвращается соответствующий ему $y_{i}$. Если $D o m=\{0,1\}^{n}$ или множество $R n g=\{0,1\}^{k}$, то соответствующие обозначения в индексе заменяются на $n$ и $k$ соответственно, т. е. вместо $\mathcal{F}_{D o m, R n g}$ пишется $\mathcal{F}_{n, k}$.

Модель идеального шифра. Для любых натуральных $n$ и $k$ обозначим

$$
\begin{aligned}
B C(k, n)=\left\{E:\{0,1\}^{n} \times\{0,1\}^{k} \rightarrow\{0,1\}^{n}: \forall K \in\{0,1\}^{k}\right. \\
\left.E(\cdot, K) \text { является биективным на множестве }\{0,1\}^{n}\right\} .
\end{aligned}
$$

Идеальным шифром называется идеальный примитив $C_{k, n}=(E, D)$ с двумя интерфейсами, реализующими соответственно преобразование $E(x, k)=y$, случайно выбранное из $B C(k, n)$, и обратное ему преобразование $D(y, k)=x$ (см. [5, с. 7]).

Хэш-функции. Пусть Dom $\subseteq\{0,1\}^{*}-$ непустое множество строк, a Rng - некоторое непустое множество (обычно $\{0,1\}^{n}$ ). Хэщ-функиией называется алгоритм, который вычисляет отображение $H: D o m \rightarrow R n g$. В данной статье мы будем рассматривать хэш-функции, использующие некоторый идеальный примитив $P$. Чтобы подчеркнуть эту зависимость, будем писать $H^{P}$. Для некоторых $n, d>0$ хэш-функцию $f^{P}:\{0,1\}^{n} \times\{0,1\}^{d} \rightarrow$ $\rightarrow\{0,1\}^{n}$ будем называть функиией сжатия.

Семейством хэи-функциий будем называть некоторое нумерованное множество хэш-функций $\mathcal{H}$, в котором для любого $n \in \mathbb{N}$ определена хэшфункция $H_{n} \in \mathcal{H}$, осуществляющая отображение $H_{n}: D o m \rightarrow\{0,1\}^{n}$, где $D o m \subseteq\{0,1\}^{*}$. 
О противнике. Будем называть противником для семейства хэш-функций $\mathcal{H}=\left\{H_{n} \mid H_{n}: D o m \rightarrow\{0,1\}^{n}\right\}$ эффективную по параметру $n$ вероятностную машину Тьюринга $A$, на вход которой подается параметр $n$. При рассмотрении работы противника время обращения к другим машинам Тьюринга будем считать равным одному такту работы противника. В зависимости от задачи вывод противника $A$ может различаться.

Напомним некоторые определения, которые Е. Додис, Т. Ристенпарт и Т. Шримптон ввели в работе [5].

Устойчивость к коллизиям (CR). Зафиксируем множество Dom $\subseteq$ $\subseteq\{0,1\}^{*}$, и пусть $A-$ противник, выводящий две строки: $x, x^{*} \in D o m$ (CRпротивник). Пусть $P$ - идеальный примитив. Для семейства хэш-функций $\mathcal{H}^{P}=\left\{H_{n}^{P} \mid H_{n}^{P}: D o m \rightarrow\{0,1\}^{n}\right\}$ и противника $A C R$-преимущчество определяется как

$$
A d v_{H_{n}^{P}, P}^{c r}(A)=\operatorname{Pr}\left[\left(x, x^{*}\right) \stackrel{\$}{\models} A^{P}: H_{n}^{P}(x)=H_{n}^{P}\left(x^{*}\right) \wedge x \neq x^{*}\right] .
$$

Если $A d v_{H_{n}^{P}, P}^{c r}(A)$ как функция от $n$ достаточно мало для любого противника $A$, то семейство функций $\mathcal{H}^{P}$ называется $C R$-защчищеннылм, или yстойчивым к коллизиям. В понимании данного определения может помочь рисунок 1 .

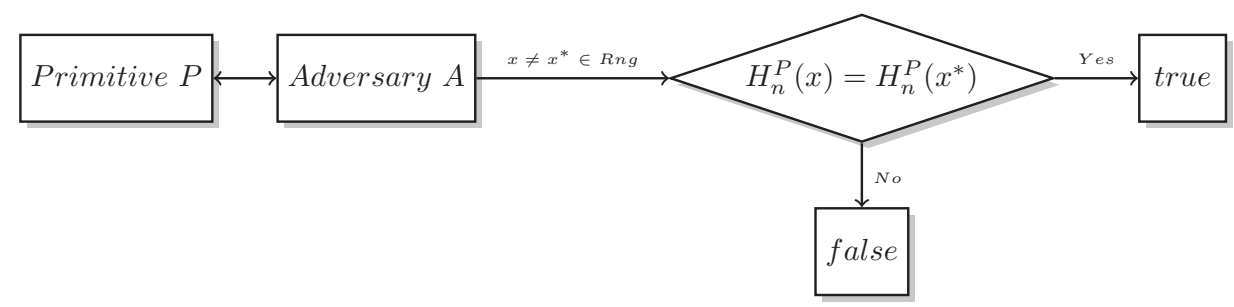

Рис. 1. Устойчивость к коллизиям.

Неотличимость. Пусть $\hat{\mathcal{H}}^{P}-$ семейство хэш-функций, которое использует идеальный примитив $P$ и отображает множество $D o m$ в множество $\{0,1\}^{n}$, а $\mathcal{H}^{Q}$ - другое семейство хэш-функций, которое использует идеальный примитив $Q$ и также отображает множество $D o m$ в множество $\{0,1\}^{n}$. Семейство $\hat{\mathcal{H}}^{P}$ называется неотличимым (indifferentiable) от $\mathcal{H}^{Q}$, если существует такой симулятор (MT) $S$, симулирующий поведение $P$, который может делать запросы к $Q$, что для любого противника $A$, выводящего 1 или 0 
(PRO-противника), indiff-преимущество

$$
A d v_{\hat{H}_{n}^{P}, H_{n}^{Q}, S}^{\text {indiff }}(A)=\left|\operatorname{Pr}\left[A^{\hat{H}_{n}^{P}, P} \Rightarrow 1\right]-\operatorname{Pr}\left[A^{H_{n}^{Q}, S} \Rightarrow 1\right]\right|
$$

как функция от $n$ достаточно мало для любого противника $A$. Если преимущество $A d v_{\hat{H}_{n}^{P}, H_{n}^{Q}, S}^{\text {indiff }}(A)$ достаточно мало, то можно использовать $\hat{\mathcal{H}}^{P}$ вместо $\mathcal{H}^{Q}$ с точностью до оценки неотличимости.

PRO-защищенность (неотличимость от случайного оракула) семейства хэш-функций $\mathcal{H}^{P}=\left\{H_{n}^{P} \mid H_{n}^{P}: D o m \rightarrow\{0,1\}^{n}\right\}$ определяется следующим образом: заменим в приведенном выше определении $\mathcal{H}^{Q}$ семейством случайных оракулов $\mathcal{F}_{D o m, n}$, т. е. положим $H_{n}^{Q}=Q=\mathcal{F}_{D o m, n}$. Определим PRO-преимущество как $A d v_{H_{n}^{P}, S}^{P R O}(A)=A d v_{H_{n}^{P}, \mathcal{F}_{D o m, n}, S}^{\text {indif }}(A)$. Иначе говоря,

$$
A d v_{H_{n}^{P}, S}^{P R O}(A)=\left|\operatorname{Pr}\left[A^{H_{n}^{P}, P} \Rightarrow 1\right]-\operatorname{Pr}\left[A^{\mathcal{F}_{D o m, n}, S} \Rightarrow 1\right]\right|,
$$

где симулятор $S$ может делать запросы к $\mathcal{F}_{D o m, n}$. Если для любого противника $A$ преимущество $A d v_{H_{n}^{P}, S}^{P R O}(A)$ достаточно мало как функция от $n$, будем называть $\mathcal{H}^{P}$ неотличимым от случайного оракула, или PRO-защищенным.

PGV-схемы первого типа. В своей работе Мартин Стэм (Martijn Stam) [6] предложил классификацию функций сжатия, основанных на использовании блочных шифров. Он заметил, что эти функции, получая на вход переменную сцепления $v \in\{0,1\}^{n}$ и блок сообщения $m \in\{0,1\}^{d}$, используют их следующим образом:

$$
\begin{aligned}
& f^{E}(v, m): \\
& (k, x) \leftarrow C^{P R E}(v, m) ; \\
& y \leftarrow E(k, x) ; \\
& \text { Ret } w \leftarrow C^{P O S T}(v, m, y),
\end{aligned}
$$

где $C^{P R E}:\{0,1\}^{n} \times\{0,1\}^{d} \rightarrow\{0,1\}^{d} \times\{0,1\}^{n}-$ функция предобработки, $E(k, x)$ - блоковый шифр, а $C^{P O S T}:\{0,1\}^{n} \times\{0,1\}^{d} \times\{0,1\}^{n} \rightarrow\{0,1\}^{n}-$ функция постобработки. Стэм определил также вспомогательную функцию постобработки $C^{A U X}(k, x, y):\{0,1\}^{d} \times\{0,1\}^{n} \times\{0,1\}^{n} \rightarrow\{0,1\}^{n}$, которая выполняет преобразование $C^{A U X}(k, x, y)=C^{P O S T}\left(C^{-P R E}(k, x), y\right)$, где $C^{-P R E}$ обозначает обратную к $C^{P R E}$ функцию, если $C^{P R E}$ является биективным отображением. В данных обозначениях предполагается существование эффективных по $n$ алгоритмов, реализующих функции $C^{P R E}, C^{-P R E}$ и $C^{P O S T}$. 
Стэм назвал функцию сжатия $P G V$-схемой типа 1, если:

1) $C^{P R E}$ является биективным отображением;

2) для любых $v, m$ отображение $C^{P O S T}(v, m, \cdot)$ является биективным;

3 ) для любых $k, y$ отображение $C^{A U X}(k, \cdot, y)$ является биективным.

Когда выполняются сформулируемые условия, через $C^{-P O S T}(v, m, \cdot)$ и $C^{-A U X}(k, \cdot, y)$ обозначим функции, обратные к $C^{P O S T}(v, m, \cdot)$ и $C^{A U X}(k, \cdot, y)$ соответственно.

Стойкость к атаке поиска прообраза. Будем использовать следующее понятие стойкости к атаке поиска прообраза [5]. Зафиксируем множество $D o m \subseteq\{0,1\}^{*}$, и пусть $A-$ противник, выводящий строку $x \in D o m$. Рассмотрим $\operatorname{Pr}\left[\mathbf{E x p}_{H, P, \mathcal{E}, A}^{p r a} \Rightarrow \operatorname{true}\right]$, где процедура $\mathbf{E x p}_{H, P, \mathcal{E}, A}^{p r a}$ описана следующей последовательностью действий:

\begin{tabular}{|c|c|c|}
\hline $\mathbf{E x p}_{H, P, \mathcal{E}, A}^{p r a}$ & oracle $\mathrm{P}(\mathrm{m}):$ & oracle $\operatorname{Ex}(z):$ \\
\hline$x \stackrel{\$}{\longleftarrow} A^{\mathrm{P}, \mathrm{Ex}}$ & $c \leftarrow P(m)$ & $Q[z] \leftarrow 1$ \\
\hline$z \leftarrow H^{P}(x)$ & $\alpha \leftarrow \alpha \|(m, c)$ & $V[z] \leftarrow \mathcal{E}(z, \alpha)$ \\
\hline $\operatorname{Ret}((x \neq V[z]) \&(Q[z]=1))$ & Ret $c$ & $\operatorname{Ret} V[z]$ \\
\hline
\end{tabular}

Противник $A$ (PrA-противник) имеет доступ к двум оракулам. Во-первых, к оракулу $P$, который предоставляет доступ к идеальному примитиву $P$ и, кроме того, сохраняет все запросы и ответы на запросы в строку состояния $\alpha$. Если $P$ предоставляет доступ к нескольким примитивам, то из строки состояния понятно, к какому именно примитиву был сделан запрос. Во-вторых, к оракулу Ех, который предоставляет доступ к экстрактору $\mathcal{E}$. Экстрактором $\mathcal{E}$ называется детерминированный алгоритм, который получает на вход точку $z \in R n g$ и строку состояния $\alpha$ и возвращает точку $x \in \operatorname{Dom} \cup\{\perp\}$, где $\perp \notin D o m-$ некоторый символ. Оракул Ех использует два массива $Q[z]$ и $V[z]$, которые в начальный момент времени для любого $z \in R n g$ равны $\perp$. Массив $Q[z]$ определяет уже запрошенные у Еx точки из $R n g$, а $V[z]-$ результат, который вернул экстрактор $\mathcal{E}$. В дальнейшем мы будем рассматривать «честные» экстракторы, то есть такие, что если $x$ является выводом экстрактора на некотором наборе $(z \in R n g, \alpha)$, то либо $x=\perp$, либо $H^{P}(x)=z$. Схематически взаимодействие машин Тьюринга можно представить в виде следующего рис. 2 .

Для семейства хэш-функций $\mathcal{H}^{P}=\left\{H_{n}^{P} \mid H_{n}^{P}: D o m \rightarrow\{0,1\}^{n}\right\}$, противника $A$ и экстрактора $\mathcal{E}$ авторы статьи [5] определяют $P R A$-преимущество 


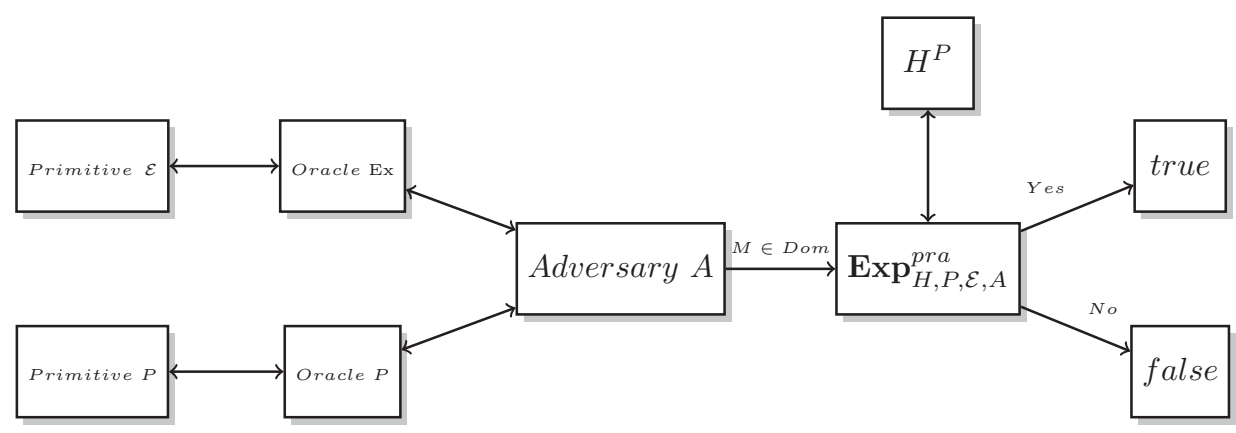

Рис. 2. Стойкость к атаке поиска прообраза.

как

$$
A d v_{H_{n}, P, \mathcal{E}}^{P r A}(A)=\operatorname{Pr}\left[\operatorname{Exp}_{H_{n}, P, \mathcal{E}, A}^{p r a} \Rightarrow \operatorname{true}\right] .
$$

Предполагается, что противник никогда не делает запрос за пределы области определения соответствующего оракула. Если существует такой эффективный по $n$ экстрактор $\mathcal{E}$, что для любого возможного противника $A$ преимущество $A d v_{H_{n} P, \mathcal{E}}^{P r A}(A)$ достаточно мало как функция от $n$, то семейство хэш-функций $\mathcal{H}$ называется стойким к атаке поиска прообраза, или PrA-защищенным.

При рассмотрении данной конструкции будем называть победой PRAпротивника $A$ событие, в результате которого $\mathbf{E x p}_{H, P, \mathcal{E}, A}^{p r a} \Rightarrow \operatorname{true.~}$

\section{2. Используемые теоремы}

Свойство стойкости к атаке на прообраз является более сильным, чем свойство усточивости к коллизиям, но более слабым, чем свойство неотличимости от случайного оракула. В работе [5] показано, что, во-первых, устойчивость к коллизиям следует из стойкости к атаке поиска прообраза, а во-вторых, что случайный оракул также является стойким к атаке поиска прообраза. Более формально: имеют место следующие 4 леммы.

Лемма 1 (теорема 3.1 из [5] ( $\operatorname{PrA} \Rightarrow \mathrm{CR})$ ). Пусть $P$ - идеальный примитив и $\mathcal{H}=\left\{H_{n} \mid H_{n}:\right.$ Dот $\left.\rightarrow\{0,1\}^{n}\right\}-$ семейство хэм-функиий. Пусть $\mathcal{E}$ - некоторый экстрактор. Пусть $A-C R$-противник против $\mathcal{H}$, делающий 
всего $q_{P}$ запросов к $Р$ и выводящий сообщения длиной не более $l_{\max }$. Тогда существует такой PrA-противник $B$, что

$$
A d v_{H_{n}, P}^{c r}(A) \leqslant A d v_{H_{n}, P, \mathcal{E}}^{P r A}(B) .
$$

При этом $\operatorname{Time}(B)=\operatorname{Time}(A)+\mathcal{O}\left(q_{P}\right)+\operatorname{Time}\left(H_{n}, l_{\max }\right), B$ делает не более $q_{P}$ запросов к $Р$ и один запрос к экстрактору.

Лемма 2 (теорема 3.2 из [5] (случайный оракул $\Rightarrow \operatorname{PrA})$ ). Пусть Dom $\subseteq\{0,1\}^{*}$ u $n>0$. Пусть $P_{n}=\mathcal{F}_{\text {Dот,n. }}$. Тогда семейство хэи-функиий $\mathcal{H}=\left\{P_{n}\right\}$ является стойким к атаке поиска прообраза. В частности, существует такой экстрактор $\mathcal{E}$, что для любого противника $A$, делающего $q_{P}$ запросов к $P$ и q запросов к экстрактору $\mathcal{E}$, выполняется неравенство

$$
A d v_{H_{n}, \mathcal{E}}^{P r A}(A) \leqslant \frac{q_{e} q_{P}}{2^{n}}+\frac{q_{P}^{2}}{2^{n}} .
$$

При этом $\operatorname{Time}(\mathcal{E})=\mathcal{O}\left(q_{e}+q_{P}\right)$.

Лемма 3 (теорема 5.1 из [5] (PGV-схема типа $1 \Rightarrow \operatorname{PrA})$ ). Зафиксируем $k, n>0$, пусть $C_{k, n}=(E, D)-$ идеальный иифр и пусть $\mathcal{H}=\left\{H^{C_{k, n}}\right\}-$ семейство $P G V$-схем типа 1, основанных на блоковом иифре. Тогда существует такой экстрактор $\mathcal{E}$, что для любого противника $A$, делающего не более $q_{P}$ запросов $\kappa C_{k, n}$ и $q_{e}$ запросов $\kappa$ экстрактору $\mathcal{E}$, будет выполняться следующее неравенство:

$$
A d v_{H_{n}, C_{k, n}, \mathcal{E}}^{P r A}(A) \leqslant \frac{q_{e} q_{P}}{2^{n}-q_{P}}+\frac{q_{P}\left(q_{P}+1\right)}{2\left(2^{n}-q_{P}\right)} .
$$

При этом $\operatorname{Time}(\mathcal{E})=\mathcal{O}\left(q_{P}\left(\operatorname{Time}\left(C^{-P R E}\right)+\operatorname{Time}\left(C^{P O S T}\right)\right)\right)$.

Лемма 4 (теорема 4.1 из [5]). Пусть $P-$ идеальный примитив $и \mathcal{H}^{P}-$ семейство хэи-функичй. Пусть $R-$ идеальный примитив с двумя интерфей-

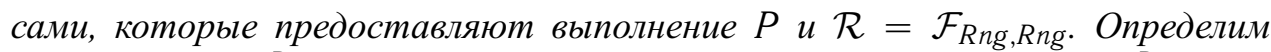
$F_{R}(M)_{n}=\mathcal{R}\left(H_{n}^{P}(M)\right)$. Пусть $\mathcal{E}-$ произвольный экстрактор для $\mathcal{H}^{P}$. Тогда существует такой симулятор (MT) $S=\left(S_{1}, S_{2}\right)$, что для каждого PROпротивника $A$, делающего не более $\left(q_{0}, q_{1}, q_{2}\right)$ запросов к оракулам $\left(F_{R}, R, S\right)$, существует такой $\operatorname{PrA-противник~} B$, что

$$
A d v_{F, R, S}^{P R O}(A) \leqslant A d v_{\mathcal{H}, P, \mathcal{E}}^{P r A}(B) .
$$

При этом Time $(S)=\mathcal{O}\left(q_{1}+q_{2} \cdot\right.$ Time $\left.(\mathcal{E})\right)$. Пусть $l_{\max }-$ максимальная длина (в битах) запроса, сделанного $A \kappa$ своему первому оракулу $F_{R}$. Противник В делает $q_{1}+q_{0} \cdot N u m Q$ ueries $\left(H, l_{\max }\right)$ запросов к идеальному примитиву $P, q_{2}$ запросов $\kappa$ экстрактору $\mathcal{E}$ и выводит строку длины не более $l_{\max }$. 
При этом Time $(B)=$ Time $(A)+\mathcal{O}\left(q_{0} \cdot \operatorname{Time}\left(H_{n}, l_{\max }\right)+q_{1}+q_{2}\right)$. Здесь под NumQueries $\left(H, l_{\max }\right)$ понимается максимальное количество обращений функиии $H$ к примитиву $P$ на входе длины не более $l_{\max }$.

\section{3. ГOCT P 34.11-2012}

\section{1. Определение}

Приведем формальное описание хэш-функции ГОСТ Р 34.11-2012 из [1]. Рассмотрим функцию сжатия

$g_{N}(v, m)=E(L \circ P \circ S(v \oplus N), m) \oplus v \oplus m \mid\{0,1\}^{512} \times\{0,1\}^{512} \rightarrow\{0,1\}^{512}$.

Здесь $L-$ умножение слева на некоторую матрицу над полем $G F(2), P-$ некоторая перестановка байт, $S$ - нелинейное биективное отображение байт.

Блочный шифр $E$ определяется следующим образом:

$$
E(k, m)=X\left[K_{13}\right] \circ \prod_{i=1}^{12} L \circ P \circ S \circ X\left[K_{i}\right](m),
$$

где $X[K](m)=m \oplus K$, а раундовые ключи вычисляются по формуле

$$
K_{i}=L \circ P \circ S\left(C_{i-1} \oplus K_{i-1}\right),
$$

$K_{0}=k, C_{i}-$ некоторые константы, описанные в [1].

Процедуру вычисления хэш-значения сообщения $M$ хэш-функцией ГОСТ Р 34.11-2012 можно описать с использованием следующего алгоритма GOST:

$$
\begin{aligned}
& \text { GOST } \\
& M_{1} \leftarrow M\|1\| 0^{511-(\text { Len mod } 512)} \| \text { Len } \| \text { Sum } \\
& m_{1}, m_{2}, \ldots, m_{k} \longleftarrow M_{1} \\
& N=0 ; v_{1}=I V \\
& \text { for } i=1 \text { to } k-2 \text { do } \\
& \quad v_{i+1}=g_{N}\left(v_{i}, m_{i}\right) \\
& \quad N=N+512 \\
& v_{k}=g_{0}\left(v_{k-1}, m_{k-1}\right) \\
& v_{k+1}=g_{0}\left(v_{k}, m_{k}\right) \\
& \text { return } v_{k+1}
\end{aligned}
$$


Здесь через Len $\in\{0,1\}^{512}$ обозначена длина сообщения $M$, а через Sum $\in\{0,1\}^{512}$ - сумма блоков длины 512 и оставшегося блока длиной $|M|-(k-3) \cdot 512$ сообщения $M$ по модулю $2^{512}$, при этом используется естественное взаимно-однозначное соответствие элементов множества $\{0,1\}^{512}$ и кольца вычетов $\mathbb{Z}_{2512}$.

Данная процедура при значении $I V=0^{512}$ реализует алгоритм хэшфункции ГОСТ Р 34.11-2012 с длиной хэш-кода 512 бит. Для получения длины хэш-кода 256 бит используется значение инициализационного вектора $I V=(00000001)^{64}$, а вместо значения $v_{k+1}$ выдаются его старшие 256 бит.

\section{2. Построение семейства хэш-функций $\mathcal{G O S T}$}

Определим семейство хэш-функций $\mathcal{G O S T}$, построенное на основе функции ГОСТ Р 34.11-2012. Для функций $G O S T_{512} \in \mathcal{G O S T}$ и $G O S T_{256} \in \mathcal{G O S T}$ положим их равными процедуре GOST с соответствующим размером выходного сообщения и значением $I V$, при условии замены функции $E$ идеальным шифром. Для остальных натуральных $n$ для построения функций $G O S T_{n} \in \mathcal{G O S T}$ рассмотрим семейство функций сжатия

$\left\{g_{N}(v, m)_{n}\right\}=\left\{E\left(L_{n} \circ P_{n} \circ S_{n}(v \oplus N), m\right) \oplus v \oplus m \mid\{0,1\}^{n} \times\{0,1\}^{n} \rightarrow\{0,1\}^{n}\right\}$,

где $L_{n}, P_{n}, S_{n}$ - некоторые биективные преобразования на $\{0,1\}^{n}$, а $E-$ функция, реализуемая первым интерфейсом идеального шифра, отображающая $\{0,1\}^{n} \times\{0,1\}^{n} \rightarrow\{0,1\}^{n}$. На основе этого построим алгоритм, реализующий функцию $G O S T_{n}:\{0,1\}^{*} \rightarrow\{0,1\}^{n}$ :

$$
\begin{aligned}
& \text { GOST } \\
& \hline M_{1} \leftarrow M\|1\| 0^{n-1-(\text { Len } \bmod n)} \| \text { Len } \| \text { Sum } \\
& m_{1}, m_{2}, \ldots, m_{k} \leftarrow M_{1} \\
& N=0 ; v_{1}=I V \\
& \text { for } i=1 \text { to } k-2 \text { do } \\
& \quad v_{i+1}=g_{N}\left(v_{i}, m_{i}\right)_{n} \\
& \quad N=N+n \\
& v_{k}=g_{0}\left(v_{k-1}, m_{k-1}\right)_{n} \\
& v_{k+1}=g_{0}\left(v_{k}, m_{k}\right)_{n} \\
& \text { return } v_{k+1}
\end{aligned}
$$

Здесь через Len $\in\{0,1\}^{n}$ обозначена длина сообщения $M$, а через Sum $\in\{0,1\}^{n}$ - сумма блоков длины $n$ и оставшегося блока длиной $|M|-$ $-(k-3) \cdot n$ сообщения $M$ по модулю $2^{n}$, при этом используется естественное 
взаимно-однозначное соответствие элементов множества $\{0,1\}^{n}$ и кольца вычетов $\mathbb{Z}_{2512}$. Сообщение $M_{1}$ в дальнейшем будем называть дополнением сообщения $M$. Схематически процесс вычисления функции $G O S T_{n}$ можно описать рисунком 3.

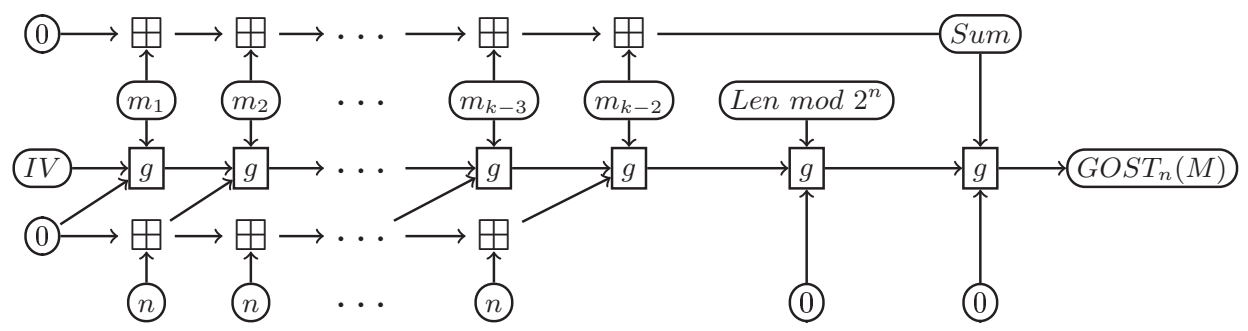

Рис. 3. Вычисление функции $G O S T_{n}$.

\section{3. Стойкость $\mathcal{G O S T}$ к атаке поиска прообраза и атаке поиска коллизий}

Покажем, что семейство хэш-функций $\mathcal{G O S T}$ является стойким к атаке поиска прообраза.

Функция $g_{0}(\tau, m)_{n}$ удовлетворяет следующим условиям:

- $C^{P R E}(v, m)=\left(L_{n} \circ P_{n} \circ S_{n}(v), m\right)$ является биективным отображением;

- $C^{P O S T}(v, m, y)=y \oplus v \oplus m$ является биективным отображением относительно переменной $y$;

- $C^{A U X}(k, x, y)=y \oplus x \oplus S_{n}^{-1} \circ P_{n}^{-1} \circ L_{n}^{-1}(k)$ является биективным отображением относительно переменной $x$.

Следовательно, функция сжатия $g_{0}(\tau, m)_{n}$ является PGV-схемой типа 1 , а значит, является стойкой к атаке поиска прообраза согласно лемме 3.

В дальнейшем нам также понадобится следующее свойство функции $g_{N}(\cdot, \cdot)_{n}: g_{N}(v, m)_{n}=g_{0}(v \oplus N, m)_{n} \oplus N$.

Теорема 3.1. Для любого эффективного по $n$ экстрактора $\mathcal{E}_{g}$ для семейства функций $\left\{g_{0}(v, m)_{n}\right\}$ существует такой эффективный по $n$ экстрактор $\mathcal{E}_{G}$ для семейства функиий $\mathcal{G O S T}$, что для любого PRAпротивника $A$, делающего не более $q_{p}$ запросов $к$ идеальному примитиву $E$ 
и qе запросов к экстрактору $\mathcal{E}_{G}$ и при любом п выводящего сообщение длиной не более $l_{\max }(n) \cdot n \leqslant 2^{n}$ символов, где $l_{\max }(n)$ - некоторая полиномиальная nо п функиия, существует такой PRA-противник $B$, что

$$
A d v_{G O S T_{n}, E, \mathcal{E}_{G}}^{P r A}(A) \leqslant A d v_{g_{0}(v, m)_{n}, E, \mathcal{E}_{g}}^{P r A}(B) .
$$

При этом противник $B$ делает не более $q_{p}+l_{\max }(n)+2$ запросов к идеальному примитиву $E$ и не более $q_{e} \cdot\left(l_{\max }(n)+2\right)$ запросов $к$ экстрактору $\mathcal{E}_{g}$.

Доказательство. Построим на основе экстрактора $\mathcal{E}_{g}$ экстрактор $\mathcal{E}_{G}$ для семейства функций $\mathcal{G O S T}$ :

$$
\begin{aligned}
& \quad \mathcal{E}_{G}(z, \alpha) \\
& \left(v_{1}, m_{1}\right) \leftarrow \mathcal{E}_{g}(z, \alpha) ; \\
& \text { if }\left(v_{1}, m_{1}\right)=\perp \text { then Ret } \perp ; \\
& \left(v_{2}, m_{2}\right) \leftarrow \mathcal{E}_{g}\left(v_{1}, \alpha\right) ; \\
& \text { if }\left(v_{2}, m_{2}\right)=\perp \text { then Ret } \perp ; \\
& i=3 ; N=m_{2}-\left(m_{2} \text { mod } n\right) ; \\
& \text { if } N>n \cdot\left(l_{\max }(n)+2\right) \text { then Ret } \perp \text {; } \\
& \text { while } N \geqslant 0 \text { do } \\
& \quad\left(v_{i}, m_{i}\right) \leftarrow \mathcal{E}_{g}\left(v_{i-1} \oplus N, \alpha\right) ; \\
& \quad \text { if }\left(v_{i}, m_{i}\right)=\perp \text { then } \operatorname{Ret} \perp ; \\
& \quad i=i+1 ; \\
& \quad v_{i}=v_{i} \oplus N ; \\
& \quad N=N-n ; \\
& \text { if } v_{i} \neq I V \text { then Ret } \perp ; \\
& \text { M } \leftarrow \text { unpad }\left(m_{i}\left\|m_{i-1}\right\| \ldots \| m_{1}\right) ; \\
& \text { Ret M; }
\end{aligned}
$$

Рассмотрим данный псевдокод подробнее. Экстрактор $\mathcal{E}_{G}$ принимает на вход строку $z \in\{0,1\}^{n}$ и строку состояния $\alpha$. Затем он вычисляет $\left(v_{1}, m_{1}\right) \leftarrow$ $\leftarrow \mathcal{E}_{g}(z, \alpha)$. Если $\mathcal{E}_{g}$ возвращает $\perp$, то $\mathcal{E}_{G}$ выдает $\perp$ и завершает свою работу. Если нет, то вычисляется следующее значение $\left(v_{2}, m_{2}\right) \leftarrow \mathcal{E}_{g}\left(v_{1}, \alpha\right)$. Заметим, что в случае удачной работы экстрактора $\mathcal{E}_{G}$ символ $m_{1}$ будет обозначать сумму блоков выводимого сообщения $M$, а $m_{2}$ - длину сообщения $M$. Если $\mathcal{E}_{g}$ возвращает $\perp$, то $\mathcal{E}_{G}$ выдает $\perp$ и завершает свою работу. Иначе $\mathcal{E}_{G}$ вычисляет $N=m_{2}-\left(m_{2} \bmod n\right)$ и запускает цикл, в котором последовательно вычисляет $\left(v_{i}, m_{i}\right)$ с помощью свойства $g_{N}(h, m)=g_{0}(h \oplus N, m) \oplus N$ и экстрактора $\mathcal{E}_{g}$ функции сжатия $g_{0}$. Если в какой-то момент $\mathcal{E}_{g}$ возвращает $\perp$, то $\mathcal{E}_{G}$ выдает $\perp$ и завершает свою работу. В противном случае $\mathcal{E}_{G}$ по окончании цикла запускает функцию $\operatorname{unpad}\left(m_{i}|| m_{i-1}\|\ldots\| m_{1}\right)$, которая проверяет, 
могло ли получиться сообщение $\hat{M}=\left(m_{i}|| m_{i-1}\|\ldots\| m_{1}\right)$ из некоторого сообщения $M$ в результате процедуры дополнения (padding) входного сообщения, то есть $\hat{M}=\left(M\left\|1|| 0^{n-1-(\text { Len mod } n)}|| \operatorname{Len}\right\|\right.$ Sum $)$, и возвращает $M$, если могло, и $\perp$, если нет. По завершении работы функции unpad $(\hat{M})$ экстрактор $\mathcal{E}_{G}$ возвращает результат ее работы. Экстрактор $\mathcal{E}_{G}$ является эффективным по $n$ алгоритмом, так как производит не более $l_{\max }(n)+2$ полиномиальных по $n$ действий в цикле и полиномиален по $n$ вне цикла. Первое утверждение следует из ограничения $N \geqslant n \cdot\left(l_{\max }(n)+2\right)$ (в противном случае не будет выполняться условие входа в цикл), второе очевидно из построения $\mathcal{E}_{G}$.

Теперь построим противника $B$ (псевдокод 2 ), используя противника $A$ и процедуру $\operatorname{Sim}_{G}$ (псевдокод 1). В дальнейшем оракулы $\operatorname{Ex}_{A}$ и $\operatorname{Ex}_{B}-$ это оракулы экстракторов, возникающие в экспериментах $\operatorname{Exp}_{G O S T_{n}, E, \mathcal{E}_{G}, A}^{p r a}$ и $\mathbf{E x p}_{g_{0}, E, \mathcal{E}_{g}, B}^{p r a}$ соответственно.

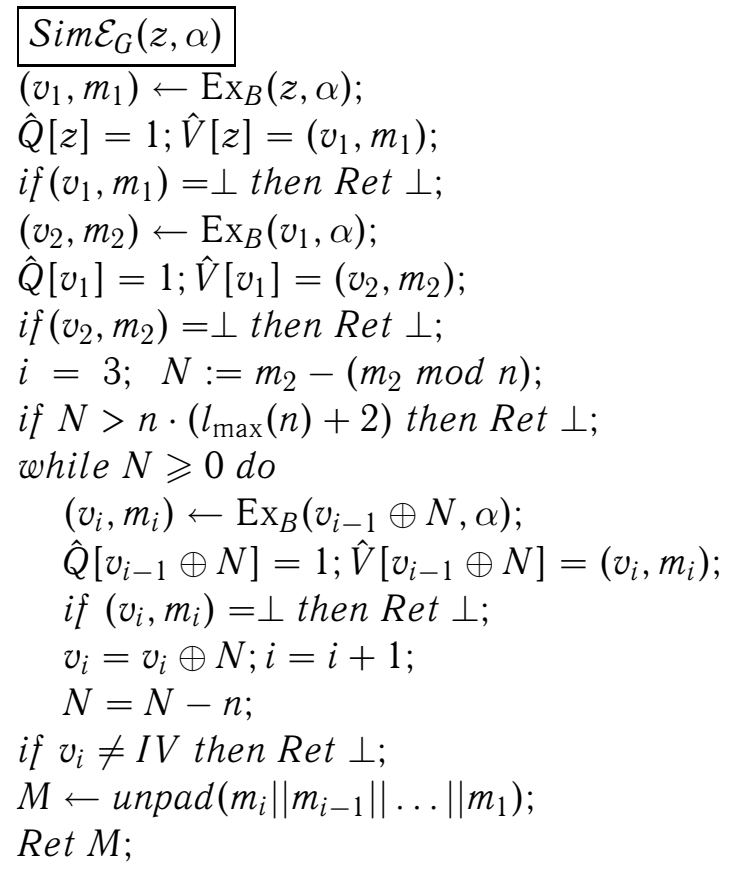

Псевдокод 1. Процедура $\operatorname{Sim}_{G}$

Рассмотрим подробнее устройство противника $B$ и процедуры $\operatorname{Sim}_{G}$. Сначала $B$ запускает противника $A$, перенаправляя его запросы к оракулу экстрактора $\operatorname{Ex}_{A}$ на вход процедуре $\operatorname{Sim}_{G}$. Процедура $\operatorname{Sim}_{G}$ действует так же, как и экстрактор $\mathcal{E}_{G}$, за исключением того, что все запросы к экстрактору $\mathcal{E}_{g}$ 


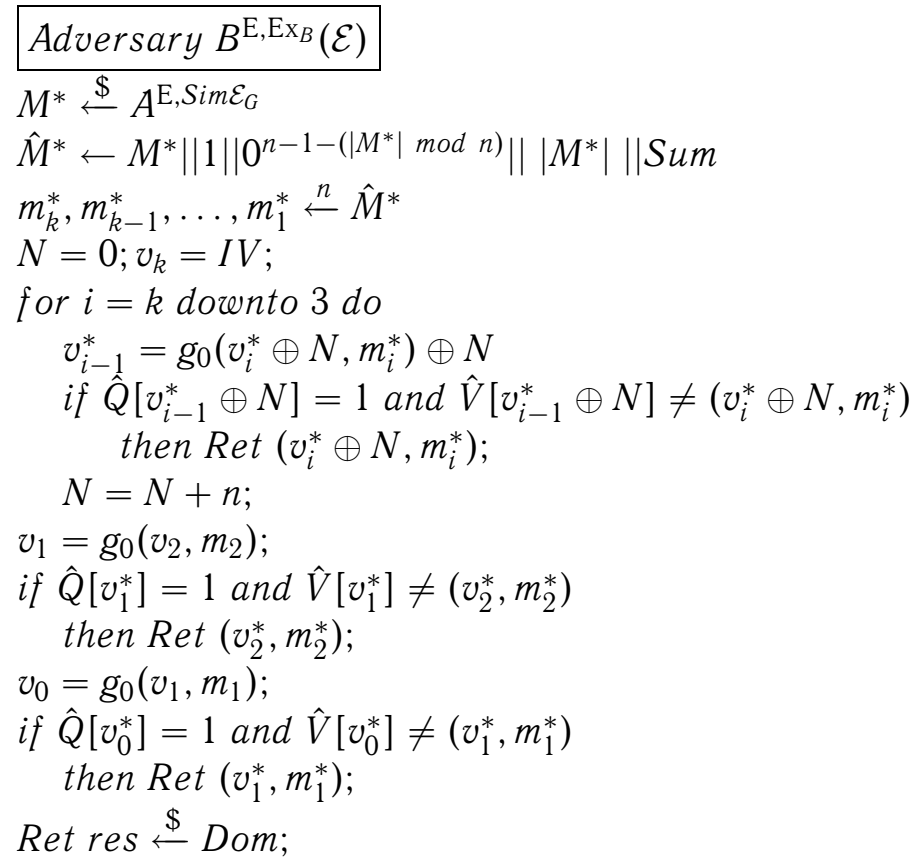

Псевдокод 2. Противник $B$

заменяются запросами к оракулу $\mathrm{Ex}_{B}$, a результат каждого запроса к этому оракулу сохраняется в массивах $\hat{Q}[$ ] и $\hat{V}[$ ]. Затем $B$ производит хэширование выведенного противником $A$ сообщения, после каждого обращения к функции сжатия проверяя истинность вывода процедуры $\mathbf{E x p}_{g_{0}, E, \mathcal{E}_{g}, B}^{p r a}$. Докажем теперь, что $A d v_{G O S T_{n}, E, \mathcal{E}_{G}}^{P r A}(A) \leqslant A d v_{g_{0}(v, m)_{n}, E, \mathcal{E}_{g}}^{P r A}(B)$.

Обозначим через $\Omega$ событие, при котором $A^{E, \mathcal{E}_{G}}$ выведет сообщение, приводящее к его победе, то есть $\Omega=\left\{\mathbf{E x p}_{G O S T_{n}, E, \mathcal{E}_{G}, A}^{p r a} \Rightarrow \operatorname{true}\right\}$. Из построения процедуры $\operatorname{Sim}_{G}$ и определения стойкости к атаке поиска прообраза следует, что

$$
\begin{aligned}
& \operatorname{Pr}[\Omega]=\operatorname{Pr}\left[\mathbf{E x p}_{G O S T_{n}, E, \mathcal{E}_{G}, A}^{\mathrm{pra}} \Rightarrow \operatorname{true}\right]= \\
& \quad=\operatorname{Pr}\left[\mathbf{E x p}_{G O S T_{n}, E, \operatorname{Sim}_{G}, A}^{\text {pra }} \Rightarrow \operatorname{true}\right]=\operatorname{Adv} v_{G O S T_{n}, E, \mathcal{E}_{G}}^{\operatorname{Pr} A}(A) .
\end{aligned}
$$

Второе равенство в цепочке следует из того, что экстрактор $\mathcal{E}_{g}$ выдает такой же ответ, как и оракул $\mathrm{Ex}_{B}$.

Покажем, что $\operatorname{Pr}[\Omega] \leqslant A d v_{g_{0}(v, m)_{n}, E, \mathcal{E}_{g}}^{\operatorname{Pr} A}(B)$. Рассмотрим элементарное событие $\omega \in \Omega$. Так как $\omega \in \Omega$, то противник $A$ выведет некоторое сообще- 
ние $M^{*}$, приводящее к его победе, в процессе работы MT $\operatorname{Exp}_{G O S T_{n}, E, \operatorname{Sim}_{G}, A}^{\text {pra }}$ Из условия $\operatorname{Exp}_{G O S T_{n}, E, S i m \mathcal{E}_{G}, A}^{\text {pra }} \Rightarrow \operatorname{true~следует,~что~} G O S T\left(M^{*}\right)$ запрашивалось у $\operatorname{Sim}_{G}$ и было возвращено такое сообщение $M$, что $M \neq M^{*}$. Тогда рассмотрим сообщения $\hat{M}=\left(m_{k}|| m_{k-1}\|\ldots\| m_{1}\right)$ и $\hat{M}^{*}=\left(m_{l}^{*}\left\|m_{l-1}^{*}\right\| \ldots \| m_{1}^{*}\right)-$ дополнения сообщений $M$ и $M^{*}$ соответственно. Из определения семейства хэш-функций $\mathcal{G O S T}$ следует, что $k>2$ и $l>2$. Докажем, что существует такое $s \leqslant \min \{k, l\}$, что $m_{s}^{*} \neq m_{s}$. Пусть данное условие не выполняется. Тогда $m_{1}^{*}=m_{1}$ и $m_{2}^{*}=m_{2}$, т. е. длины сообщений $M$ и $M^{*}$ равны, следовательно, $\min \{k, l\}=k=l$. Так как по предположению для каждого $i \leqslant \min \{k, l\}$ имеет место равенство $m_{i}=m_{i}^{*}$, то сообщения $M$ и $M^{*}$ совпадают, что противоречит событию $\omega$.

Так как из неравенства $m_{s}^{*} \neq m_{s}$ следует, что для любого $v \in\{0,1\}^{n}$ имеет место неравенство $\left(v, m_{s}^{*}\right) \neq\left(v, m_{s}\right)$, то существует такое $1 \leqslant s<3$, что $\left(v_{s}^{*}, m_{s}^{*}\right) \neq\left(v_{s}, m_{s}\right)$, или существует такое $3 \leqslant s \leqslant \min \{k, l\}$, что $\left(v_{s}^{*} \oplus N, m_{s}^{*}\right) \neq\left(v_{s}, m_{s}\right)$. Пусть $r-$ максимальное из таких значений $s$. Если $v_{r-1}^{*}=v_{r-1}$, то возврат пары $\left(v_{r}^{*} \oplus N, m_{r}^{*}\right)$ обеспечивает истинность вывода процедуры $\operatorname{Exp}_{g_{0}, E, \mathcal{E}_{g}, B}^{p r a}$, потому что $v_{r-1}$ запрашивалось у $\operatorname{Ex}_{B}$ и было возвращено значение $\left(v_{r}, m_{r}\right)$, а $\left(v_{r}^{*} \oplus N, m_{r}^{*}\right) \neq\left(v_{r}, m_{r}\right)$, хотя $g_{0}\left(v_{r}, m_{r}\right)_{n}=$ $=g_{0}\left(v_{r}^{*} \oplus N, m_{r}^{*}\right)_{n}$. Если же нет, то рассмотрим следующую пару с $r=s-$ - 1. Тогда $\left(v_{s-1}^{*} \oplus N, m_{s-1}^{*}\right) \neq\left(v_{s-1}, m_{s-1}\right)$. Если $v_{s-2}^{*}=v_{s-2}$, то возврат пары $\left(v_{s-1}^{*} \oplus N, m_{s-1}^{*}\right)$ снова обеспечивает победу $B$. В противном случае продолжаем уменьшать индекс $r$ и рассматривать пары $\left(v_{r}, m_{r}\right)$. В какой-то момент $v_{r}^{*}=v_{r}$ (в противном случае $\operatorname{GOST}(M) \neq G O S T\left(M^{*}\right)$, так как $v_{0}^{*}=$ $=\operatorname{GOST}\left(M^{*}\right)$, а $\left.v_{0}=\operatorname{GOST}(M)\right)$. Тогда возврат пары $\left(v_{r+1}^{*} \oplus N, m_{r+1}^{*}\right)$ для $r>1$ или пары $\left(v_{r+1}^{*}, m_{r+1}^{*}\right)$ для $r \leqslant 1$ обеспечивает победу $B$. Изменения в случае $r \leqslant 1$ связаны с тем, что последние два блока соответствующих сообщений обрабатываются функцией сжатия $g_{0}(v, m)_{n}$, а не $g_{N}(v, m)_{n}$. Отсюда получаем, что

$$
A d v_{g, E, \mathcal{E}_{g}}^{P r A}(B) \geqslant \operatorname{Pr}[\omega]=A d v_{G, E, \mathcal{E}_{G}}^{\operatorname{Pr} A}(A) .
$$

Теперь докажем эффективность по $n$ построенной нами МТ $B$. Противник $B$ состоит из двух частей. Первая - это запуск противника $A$. Так как $A$ по определению противника является эффективным по $n$ алгоритмом, то эта часть эффективна по $n$. В ходе запуска противника $A$ происходит не более $q_{p}$ запросов к идеальному примитиву $E$ и не более $q_{e} \cdot\left(l_{\max }(n)+2\right)$ запросов к экстрактору $\mathcal{E}_{g}$, так как при каждом запросе противника $A$ к процедуре $\operatorname{Sim}_{G}$ происходит не более $\left(l_{\max }(n)+2\right)$ запросов к $\operatorname{Ex}_{B}$. Иначе противник $A$ мог бы возвращать сообщение длиной более $l_{\max }(n) \cdot n$, что противоречит условию теоремы. Во второй части МТ $B$ производит хэширование полученного на 
первом этапе сообщения $M^{*}$ длины не более $l_{\max }(n) \cdot n$. Для преобразования с помощью функции сжатия одного блока сообщения и одного вспомогательного блока требуется один запрос к идеальному примитиву $E$. Так как длина сообщения $M^{*}$ не больше $l_{\max }(n) \cdot n$, то хэширование потребует не более $\left(l_{\max }(n)+2\right)$ запросов к идеальному примитиву $E$. Так как противник $A$ может выводить сообщения не более чем полиномиальной по $n$ длины, то и вторая часть $B$ полиномиальна по $n$. Отсюда получаем полиномиальность противника $B$ по $n$, что и завершает доказательство.

Теорема 3.2 (ГОСТ является PrA-защищенным). Семейство хэшфункиий $\mathcal{G O S T}$ является стойким $к$ атаке поиска прообраза и к атаке поиска коллизий.

Доказательство. Согласно определению $\operatorname{PrA-защищенности~требу-~}$ ется показать, что существует такой эффективный экстрактор $\mathcal{E}$, что для любого возможного противника $A$ преимущество $A d v_{G O S T_{n}, E, \mathcal{E}}^{P r A}(A)$ достаточно мало как функция от $n$.

Так как функция $g_{0}(v, m)_{n}$ является PGV-схемой типа 1 , а следовательно, и $\operatorname{PrA-защищенной,~то~для~нее~существует~такой~экстрактор~} \mathcal{E}_{g}$, что для любого возможного противника $B$ преимущество $A d v_{g_{0}(v, m)_{n}, E, \mathcal{E}_{g}}(B)$ достаточно мало как функция от $n$. Зафиксируем данный экстрактор $\mathcal{E}_{g}$. Согласно теореме 3.1 существует такой экстрактор $\mathcal{E}_{G}$ для функции $G O S T_{n}$, что для любого противника $A$, делающего не более $q_{p}$ запросов к идеальному примитиву $E$ и $q_{e}$ запросов к экстрактору $\mathcal{E}_{G}$ и выводящего сообщение длины не более $l_{\max }(n) \cdot n \leqslant 2^{n}$ символов, где $l_{\max }(n)$ - некоторая полиномиальная по $n$ функция, существует такой противник $B$, что

$$
A d v_{G O S T_{n}, E, \mathcal{E}_{G}}^{P r A}(A) \leqslant A d v_{g_{0}(v, m)_{n}, E, \mathcal{E}_{g}}^{\operatorname{Pr}}(B) .
$$

Причем противник $B$ делает не более $q_{p}+l_{\max }(n)+2$ запросов к идеальному примитиву $E$ и не более $q_{e} \cdot\left(l_{\max }+2\right)$ запросов к экстрактору $\mathcal{E}_{g}$.

По лемме 3 преимущество $A d v_{g_{0}(v, m)_{n}, E, \mathcal{E}_{g}}^{P r A}(B)$ может быть ограничено сверху в терминах числа запросов противника $A$ к экстрактору $\mathcal{E}_{G}$ из теоремы 3.1 и идеального примитива $E$, то есть

$$
\begin{aligned}
& A d v_{g_{0}(v, m)_{n}, E, \mathcal{E}_{g}}^{P r A}(B) \leqslant \frac{q_{e}\left(l_{\max }+2\right)\left(q_{p}+l_{\max }(n)+2\right)}{2^{n}-\left(q_{p}+l_{\max }(n)+2\right)}+ \\
& +\frac{\left(q_{p}+l_{\max }(n)+2\right)\left(q_{p}+l_{\max }(n)+3\right)}{2\left(2^{n}-\left(q_{p}+l_{\max }(n)+2\right)\right)} .
\end{aligned}
$$


Отсюда следует, что существует такой экстрактор $\mathcal{E}_{G}$ для функции $G O S T_{n}$, что для любого противника $A$, делающего максимум $q_{p}$ запросов к идеальному примитиву $E$ и $q_{e}$ запросов к экстрактору $\mathcal{E}_{G}$ и выводящего сообщение длиной не более $l_{\max }(n) \cdot n \leqslant 2^{n}$ символов, где $l_{\max }(n)$ - некоторая полиномиальная от $n$ функция, выполняется

$$
\begin{aligned}
& A d v_{G O S T_{n}, E, \mathcal{E}_{G}}^{P_{r}}(A) \leqslant \frac{q_{e}\left(l_{\max }+2\right)\left(q_{p}+l_{\max }(n)+2\right)}{2^{n}-\left(q_{p}+l_{\max }(n)+2\right)}+ \\
& +\frac{\left(q_{p}+l_{\max }(n)+2\right)\left(q_{p}+l_{\max }(n)+3\right)}{2\left(2^{n}-\left(q_{p}+l_{\max }(n)+2\right)\right)} .
\end{aligned}
$$

Следовательно, преимущество $A d v_{G O S T_{n}, E, \mathcal{E}_{G}}^{P r}(A)$ достаточно мало как функция от $n$, а значит, семейство хэш-функций $\mathcal{G O S T}$ является стойким к атаке поиска прообраза. Стойкость к атаке поиска коллизий следует из утверждения леммы 1, которое ограничивает сверху CR-преимущество PrA-преимуществом.

Применяя лемму 4 к теореме 3.2 , получаем, что семейство хэшфункций $\left\{\mathcal{F}_{n, n}\left(G O S T_{n}(M)\right)\right\}$ является неотличимым от случайного оракула произвольной длины.

\section{4. Заключение}

В работе на основе семейства хэш-функций ГОСТ Р 34.11-2012 описано такое семейство хэш-функций $\mathcal{G O S T}$, что при одинаковой длине хэш-кода функции семейства $\mathcal{G O S T}$ получаются из функций семейства ГОСТ Р 34.11-2012 заменой внутреннего шифра на идеальный. Для остальных функций семейства $\mathcal{G O S T}$ использована схожая конструкция. Для построенного семейства $\mathcal{G O S T}$ доказаны стойкость к атаке поиска прообраза и устойчивость данного семейства к коллизиям. Данные свойства семейства $\mathcal{G O S T}$ косвенно подтверждают стойкость использованной в ГОСТ Р 34.11-2012 конструкции. В дальнейшем планируется исследование семейства хэш-функций $\mathcal{G O S T}$ на соответствие свойству неотличимости от случайного оракула, а также, возможно, исследование на удовлетворение понятиям стойкости к атаке поиска прообраза и устойчивости к коллизиям семейства хэш-функций, лучше отображающего свойства функции сжатия, используемой в ГОСТ Р 34.11-2012.

\section{Благодарности}

В заключение автор выражает глубокую благодарность своему научному руководителю Карпунину Г. А. за постановку задачи и внимание к работе. 
Также автор признателен рецензенту И. В. Лаврикову за важные замечания, способствовавшие улучшению изложения результатов.

\section{Список литературы}

[1] ГОСТ Р 34.11-2012, Национальный стандарт Российской Федерации. Информаџионная технология. Криптографическая защита информаџии. Функиия хэширования., Стандартинформ, Москва, 2012.

[2] Mendel F., Pramstaller N., Rechberger C., Kontak M., Szmidt J., "Cryptanalysis of the GOST hash function CRYPTO'2008”, Lect. Notes Comput. Sci, 5157 (2008), 162-178.

[3] AlTawy R., Kircanski A., Youssef A. M., "Rebound attacks on Stribog", $I A C R$ Cryptology ePrint Archive, 2013, 539.

[4] Wang Z., Yu H., Wang X., "Cryptanalysis of GOST R hash function", IACR Cryptology ePrint Archive, 2013, 584.

[5] Dodis Y., Ristenpart T., Shrimpton T., "Salvaging Merkle-Damgård for practical applications", IACR Cryptology ePrint Archive, 2009, 177.

[6] Stam M., "Blockcipher based hashing revisited", IACR Cryptology ePrint Archive, 2008, 71 .

[7] AlTawy R., Youssef A. M., "Preimage attacks on reduced-round Stribog", IACR Cryptology ePrint Archive, 2014, 319.

[8] AlTawy R., Youssef A. M., "Watch your constants: malicious Streebog", IACR Cryptology ePrint Archive, 2014, 879.

[9] Ma B., Li B., Hao R., Li X., "Improved cryptanalysis on reduced-round GOST and Whirlpool hash function (Full version)", IACR Cryptology ePrint Archive, 2014, 375 . 